\title{
Are Exercise and Mitochondrial Antioxidants Compatible in the Treatment of Invasive Breast Cancer?
}

\author{
Jorming Goh ${ }^{1,2}$, Christina Pettan-Brewer ${ }^{1}$, Linda Enns ${ }^{1}$, Sy Fatemie ${ }^{1}$ and Warren Ladiges ${ }^{1 *}$ \\ ${ }^{1}$ Department of Comparative Medicine, University of Washington, Seattle, WA, USA \\ ${ }^{2}$ Interdisciplinary Program in Nutritional Sciences, University of Washington, Seattle, WA, USA
}

\begin{abstract}
Breast cancer is now the leading cause of cancer mortality among women worldwide, with more that 40,000 American women dying from the disease annually. While these statistics are grim, numerous epidemiological studies generally support a protective effect of physical activity for breast cancer. Animal data using voluntary wheel running and invasive cancer models are in line with human epidemiological data suggesting that physical exercise has antitumor affects and may be associated with an increase in reactive oxygen species (ROS). Elevated production of mitochondrial ROS ( $\mathrm{mtROS}$ ) is also associated with the promotion of tumor progression, and attenuation of oxidative stress with a mitochondrial targeted antioxidant has been shown to reduce tumor burden and metastasis in an invasive breast cancer mouse model. However, if an anti-tumor effect of running is associated with an increase in ROS, then there is a potential paradox in that any anti-oxidant activity directed to mitochondria might mitigate ROS and prevent anti-tumor affects. There are several mechanistic scenarios involving the tumor microenvironment and tumor associated macrophages where exercise and antioxidants may be compatible, thereby suggesting that physical activity and mitochondrial antioxidants could be complimentary and/or synergistic in suppressing invasive breast cancer by preventing or reversing the pro-tumor cell microenvironment and enhancing an anti-tumor microenvironment. Investigation of oxidative stress in the tumor microenvironment is an area highly relevant to understanding not just the biology of cancer, but also the mechanisms through which regular physical activity mediates changes in normal tissue during tumorigenesis and metastasis. Several antioxidant compounds that target mitochondria, such as the SzetoSchiller (SS) peptides and mitoQ compounds, are being developed which could easily be tested in preclinical studies for compatibility with exercise training in the treatment and possible prevention of invasive breast cancer.
\end{abstract}

Keywords: Invasive breast cancer; Physical activity; ROS; Mitochondrial antioxidants; Exercise training; Tumor associated macrophages; Tumor cell microenvironment

\section{Introduction}

In the last few years, regular exercise and physical activity have garnered excitement in the field of cancer prevention. There have been consistent reports in the literature that exercise stimulates an anti-inflammatory response $[1,2]$ and upregulates gene expression of endogenous anti-oxidants [3]. These two mechanisms are potential anti-carcinogenic effects of regular exercise, since increased inflammation and mitochondrial oxidative stress are characteristic hallmarks of malignancy. In addition, a recent report using National Health and Nutrition Examination Survey (NHANES) data from 2003 to 2006 documented that $50 \%$ of Americans consume some kind of nutritional supplement, with multi-vitamins and multi-minerals the most commonly consumed supplement [4]. This social trend presents an interesting paradigm: would combined use of antioxidants and regular exercise have a synergistic effect on attenuating cancer risk? Multi-vitamins such as Vitamin $\mathrm{E}$ and $\mathrm{C}$ quench free radical activity, but are spatially restricted to the cytoplasm and unable to penetrate the mitochondria. This is where developments in mitochondrialtargeted antioxidants such as the SS peptides or mitoQ present an advantage over conventional dietary antioxidants. In this review, we describe the effects of exercise and physical activity on breast cancer outcomes and the mechanistic relationships with mitochondrial ROS. We also hypothesize the effects of combining mitochondrial-targeted antioxidants with exercise on chemoprevention.

\section{Breast cancer and physical activity}

Breast cancer is now the leading cause of cancer mortality among women worldwide. According to the American Cancer Society, there were an estimated 207,090 new cases of breast cancer in 2010, with a further 39,840 American women dying from the disease. While these statistics are grim, epidemiological evidence supports a protective effect of physical activity for breast cancer. For example, the risk of death from invasive breast carcinoma was 30\% lower in American women aged 3564 years that participated in recreational physical activity throughout their lifetime compared with women that were sedentary [5]; women with stage I-III breast cancer who participated in 3-5 hours of walking per week had decreased risk of breast cancer recurrence and mortality [6]. Moderate physical exercise, including brisk walking, reduced postmenopausal breast cancer risk suggesting that increases in activity after menopause are beneficial [7]. In 670 women diagnosed with local or regional breast cancer and monitored for six years, any recreational physical activity and consumption of better quality diets was associated with a $91 \%$ reduced risk of death from breast cancer [8]. However, not all studies have shown this negative association between increased physical activity and reduced breast cancer risk and mortality. For example, a negative correlation between amount of physical activity and risk of breast cancer mortality was recently reported [9]. The limitation of physical activity-focused epidemiological studies is that they are observational. Therefore, in order to define the underlying

*Corresponding author: Warren Ladiges DVM, Professor, Department of Comparative Medicine, School of Medicine, University of Washington, USA, Tel: (206) 685-0149; Fax: (206) 685-3006; E-mail: wladiges@u.washington.edu

Received November 15, 2011; Accepted December 20, 2011; Published December 27, 2011

Citation: Goh J, Pettan-Brewer C, Enns L, Fatemie S, Ladiges W (2012) Are Exercise and Mitochondrial Antioxidants Compatible in the Treatment of Invasive Breast Cancer? Bioenerg Open Access 1:101. doi:10.4172/2167-7662.1000101

Copyright: (c) 2012 Goh J, et al. This is an open-access article distributed unde the terms of the Creative Commons Attribution License, which permits unrestricted use, distribution, and reproduction in any medium, provided the original author and source are credited. 
associated with a beneficial effect of physical activity on cancer biology, pre-clinical studies would be useful [10].

We have conducted preliminary experiments in mice comparing the effects of two-months of voluntary nocturnal wheel running on mammary tumorigenesis and metastasis. We used the Polyoma Middle $\mathrm{T}$ Oncoprotein (PyMT) transgenic mouse, a widely used preclinical model to study metastatic breast cancer with a near 100 per cent metastasis to the lungs [11]. Although the PyMT gene is not expressed in human breast cancer cells, its products bind to, and activate several signaling pathways such as Src, Ras and Phosphatidylinositol (PI)-3 kinase, which are all implicated in human breast cancer. The PyMT model also demonstrates loss of estrogen and progesterone receptors, in addition to expressing Epidermal Growth Factor Receptor Family (ErbB2/Neu) during the late carcinoma stage, both of which resemble human breast cancer with poor prognosis [11]. Our running wheel system (Figure 1A) allows us to monitor the activity status of each individual mouse throughout the experiment. Each mouse is housed in an individually ventilated cage and provided fresh food pellets in a ceramic bowl twice weekly. Rotation of the wheel by the mouse transmits an electrical signal wirelessly to a hub and the number of revolutions is recorded on the Wheel Manager software (Med Associates Inc) every minute. The activity is recorded as the distance covered across time, and exported to Excel (Microsoft) as a graph (Figure 1B). Our preliminary data show PyMT mice that run between $12.6 \mathrm{~km}$ and $22 \mathrm{~km}$ daily for one month have decreased primary tumor invasiveness, as demonstrated by histopathological sections, compared to PyMT sedentary mice, which have their wheels locked in place (personal observations). These data are in line with human epidemiological data suggesting that physical exercise has anti-tumor affects.

\section{Physical activity and ROS}

It has been shown that ROS are generated during physical exercise in both animal models and humans, and that increased ROS production can overwhelm antioxidant systems and cause oxidative damage $[12,13]$. The term "hormesis" has been adopted to explain how a mild oxidative stress associated with moderate exercise-training can result in favorable adaptations that protect the body against more severe stresses that can cause disease, for example cancer [14]. Activation of redox-sensitive pathways, such as anti-oxidant enzymes, transcription factors, and metabolic proteins, by physical exercise can result in gene products that enhance antioxidant activity and protect against oxidative damage. Conceivably, these hormetic effects can occur within tumor cells or within immune surveillance mechanisms programmed to eradicate transformed and malignant cells. The question is whether a mitochondrial antioxidant in conjunction with a regular and consistent physical exercise program will attenuate ROS-mediated activation of redox-sensitive pathways and counterbalance anti-tumor effects. And if so, whether these antioxidant effects are solely observable in cancer cells or if cells within the tumor environment such as macrophages are also affected.

\section{Breast cancer and mitochondrial ROS}

Breast cancer is mainly a disease of older postmenopausal women. Aging is associated with gradual mitochondrial dysfunction that results in increases in ROS production and oxidative stress. Elevated production of mitochondrial ROS (mtROS) damages mitochondrial constituents, further impairs oxidative phosphorylation and results in an oxidative-damage/ mitochondrial dysfunction loop. Within tumor cells, increased mtROS can disrupt the delicate balance between tumor suppressors and oncogenes, stimulate epithelial-to-mesenchymal transition, and create an inflammatory environment conducive to tumor progression [15-17]. Additionally, mitochondrial dysfunction causes malignant cells to shift energy production from oxidative phosphorylation to aerobic glycolysis. This shift towards aerobic glycolysis generates ROS, and prevents cancer cells from depleting their ATP stores, thus avoiding mitochondrial- mediated apoptosis [18]. As well, the shift to aerobic glycolysis increases the intermediates that could be used for anabolic activities, such as nucleotide synthesis, protein translation and cell growth. In addition to generating mutations in mtDNA and impairing mitochondrial respiration, ROS act as direct signal transducers to alter downstream pathways in tumor growth and migration. Hydrogen peroxide $\left(\mathrm{H}_{2} \mathrm{O}_{2}\right)$ can activate Protein Kinase B (Akt) and inactivate Phosphatase and Tensin Homolog (PTEN) [19], two well-known pathways implicated in tumor progression. Further, exposure to $\mathrm{H}_{2} \mathrm{O}_{2}$ can induce MMP2 expression by human endothelial cells resulting in disengagement from the basement membrane [20], suggesting that a pro-oxidative microenvironment results in tumor and stromal cells secreting matrix metalloproteinases (MMPs) to degrade the local basement membrane. Subsequently, tumor cells can disseminate to distant organs.

Given that oxidative stress promotes tumor progression, attenuation of oxidative stress with an antioxidant should result in reduced tumor burden and metastasis. We have found that old mice expressing mitochondrial targeted catalase (mCAT) have a decreased tumor burden [21] and that PyMT transgenic mice expressing mCAT show lower percentages of aggressive mammary carcinomas and reduced pulmonary metastasis in association with decreased ROS levels in both the primary tumor and lungs [22]. The mCAT mouse is also protected from age-associated mitochondrial dysfunction and insulin resistance [23], suggesting that PyMT mice expressing mCAT have improved
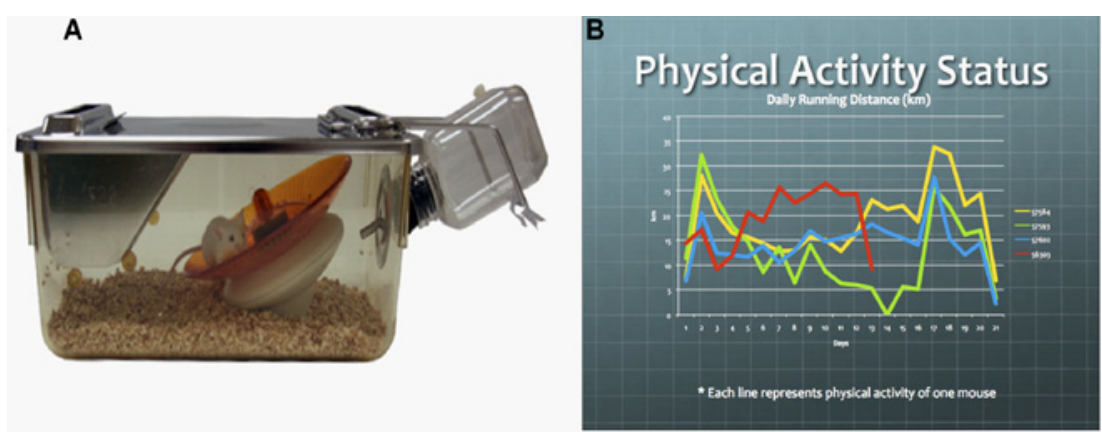

Figure 1: Voluntary running wheel setup. A. An individual mouse is given access to a running wheel that either freely rotates or is locked. Rotation of the running wheels transmits an electrical signal wirelessly to a hub and the number of revolutions is recorded on the Wheel Manager software. B. Each colored line reflects distance ran by a different mouse. 
metabolic function. Therefore, mCAT likely protects PyMT mice from metastatic progression because of changes in oxidative stress, mitochondrial function and related metabolic pathways indicating that a mitochondrial targeted antioxidant approach for breast cancer intervention may have merit.

\section{ROS and tumor associated macrophages}

Stromal cells within the tumor microenvironment secrete factors and cross-talk with cancer cells to display the phenotypic hallmarks of cancer, such as self-sufficiency in growth and increased invasiveness and metastatic potential [24]. Tumor associated macrophages (TAMs) are stromal cells generally associated with poor prognosis in cancer survivors [25]. However, TAMs are phenotypically diverse, reflecting their plasticity within different tissue microenvironments. Two different sub-populations have been described. "Classically activated", or M1 macrophages, have anti-tumor activity, secreting products that can destroy tumors by activating tumoricidal natural killer cells [26], T-helper (Th)1 cells [27], and upregulate Nuclear Factor (NF) $-\kappa B$ transcription [28] to elicit anti-tumor immune responses. "Alternatively-activated", or M2 macrophages, secrete factors and cytokines such as Vascular Endothelial Growth Factor (VEGF)-A (proangiogenic), Interleukin (IL)-10 (inhibits dendritic cell maturation and promotes Th2 response for tumor immune tolerance) and MMPs that enhances primary tumor invasiveness. It is conceivable that as a tumor is initiated to grow, M1 macrophages are recruited to the tumor microenvironment and respond in an attempt to suppress tumor growth. With progressive growth and acquisition of malignancy, tumor cell signaling polarizes M1 macrophages to differentiate towards M2 macrophages [29]. Since it has been shown that physical activity or regular exercise training can influence various aspects of macrophage physiology, such as phagocytosis, chemotaxis, metabolism and antitumor activity [30], it is possible that physical exercise could prevent $\mathrm{M} 2$ polarization and enhance the presence of M1 macrophages as an anti-tumor mechanism.

When mitochondrial-targeted antioxidants are utilized in mouse cancer models, the initial recruitment of macrophages to the tumor microenvironment during early tumorigenesis may be attenuated. This is based on our previous work showing that mCAT reduces ROS in tumor cells and cells in the tumor microenvironment [22]. We now have preliminary data to suggest that the presence of mCAT in the transgenic PyMT breast cancer mouse attenuates M2 macrophage polarization in the tumor microenvironment (personal observations). Therefore, the macrophages are still recruited to the tumor site, but the population is more M1 in polarity. As the tumor progresses, some tumor-associated macrophages would still be polarized to become M2 macrophages, but due to an attenuation of mitochondrial oxidative stress, there would be a reduction in the cytokines produced by tumor cells and/or stromal cells, resulting in a balance shifted towards M1 as opposed to M2 macrophages (Figure 2). A reduction in the M2 macrophage population would decrease the extensive basement membrane degradation and tumor invasion, and thus reduce the extent of metastasis, as well as decrease endothelial growth and vascularization [31]. When physical activity is added to the antioxidant scenario, the hypothesis is that, because exercise increases the Th1/Th2 balance in the tumor microenvironment [32], there should be an increase in M1 TAMs (Figure 2), and the addition of a mitochondrial antioxidant should further enhance the M1 TAM population.

\section{Clinical implications}

There is a potential paradox if an anti-tumor effect of running is associated with an increase in ROS in that any anti-oxidant activity might mitigate ROS and prevent anti-tumor affects. There are several mechanistic scenarios where exercise and antioxidants might be compatible. First, increases in ROS production in tumor cells may not be accompanied by increases in endogenous antioxidant activity in those tumor cells, resulting in a ROS-triggered pathway to apoptosis. Since some antioxidants may induce apoptosis in tumor cells [33], a possible mechanism for an enhanced anti-tumor affect could be accelerated apoptosis. Secondly, it is possible that physical activity in combination with a mitochondrial targeted antioxidant improves mitochondrial respiration, thus, by improving mitochondrial respiration in tumor cells, substrate utilization is shifted, and "aerobically conditioned" cells may be less able to survive than their hypoxia-conditioned counterparts. These mechanisms may act in concert and provide exciting intervention targets for breast cancer patients [34,35].

Thirdly, running may generate ROS by mechanisms other than

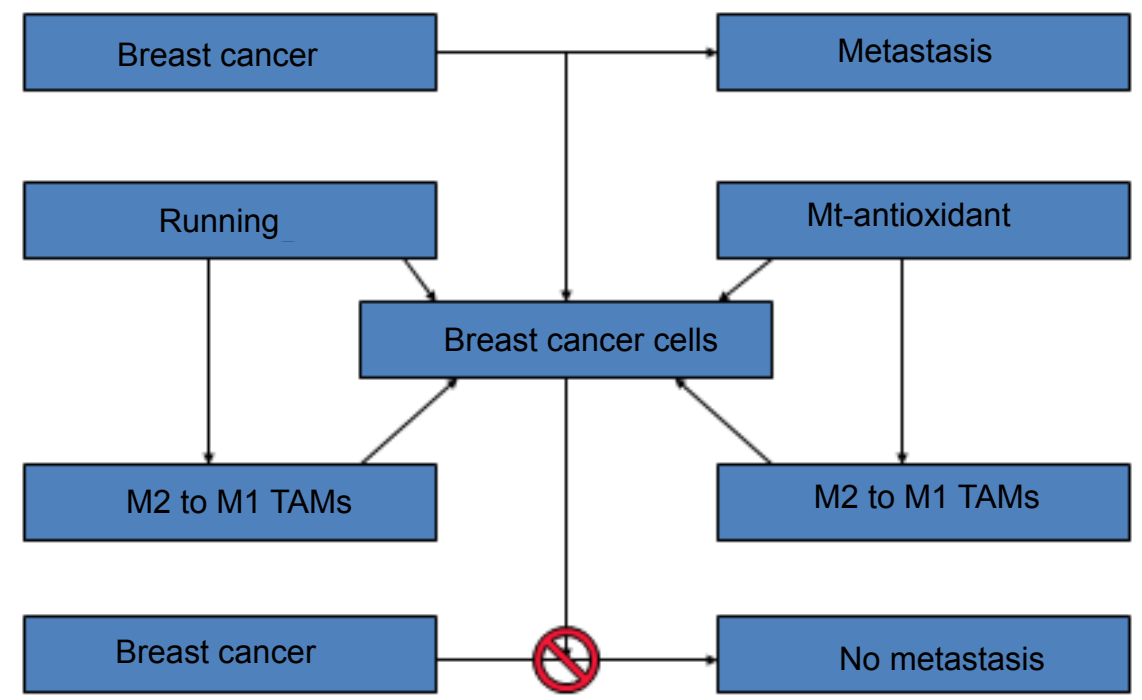

Figure 2: Physical activity and mitochondrial targeted antioxidants may be complimentary and/or synergistic in suppressing invasive breast cancer by preventing or reversing a protumor cell microenvironment (M2 TAMs) and enhancing an anti-tumor microenvironment (M1 TAMs). 
those involving mitochondria. Exercise-induced oxidative stress was originally thought to originate from mitochondria in skeletal muscle fibers, due to the increased metabolic demand or as a consequence of skeletal muscle damage [36]. Earlier support for skeletal muscle mitochondria as key sources of exercise-induced ROS was based on the fact that increased oxygen consumption during exercise could elicit a proportionate 50- to 100 -fold increase in superoxide generation [37]. However, during exercise, skeletal muscle mitochondria are primarily at a low state of respiration associated with relatively low levels of ROS [38]. In another study, skeletal muscle myotubes electrically stimulated to simulate contractile activity demonstrated only a mild increase in the rate of oxidation [39]. This relatively small increase in oxidative stress does not support mitochondria as a major source of exercise-induced ROS. There are several sources of extra-mitochondrial ROS that might contribute to exercise-induced oxidative stress including reduced Nicotinamide Adenine Dinucleotide Phosphate (NADPH)-dependent oxidase found to be located in the sarcoplasmic reticulum of skeletal muscle and capable of reducing oxygen to form super oxide [40]. A second source could be calcium-independent isoform of skeletal muscle phospholipase $\mathrm{A}_{2}\left(\mathrm{PLA}_{2}\right)$ shown to generate ROS in skeletal muscle [41]. A third source could be xanthine oxidase, shown to be associated with increased superoxide during muscle contraction and reperfusion [42]. Hence, it is plausible that exercise-induced oxidative stress and the subsequent redox-signaling pathways may be different from that of tumor-associated redox signaling and provide complementary ways of attenuating invasive cancer.

In summary, implications for human cancer intervention are huge if it can be shown that physical activity and mitochondrial antioxidants are complimentary and/or synergistic in suppressing invasive breast cancer. These affects could act directly on tumor cells and may in fact target cells in the tumor microenvironment by preventing or reversing pro-tumor support and enhancing anti-tumor activity. An example would be TAMs and the ability of an exercise and mitochondrial combination to repolarize from an M2 support mode to an M1 inhibitory mode. Oxidative stress in the tumor microenvironment has received relatively little attention in the context of physical activity and breast cancer research. This is an area highly relevant to understanding not just the biology of cancer, but also the mechanisms through which regular physical activity mediates changes in normal tissue during tumorigenesis and metastasis. Several antioxidant compounds that target mitochondria, such as the SS peptides [43] and mitoQ compounds [44], are being developed which could easily be tested in preclinical studies for compatibility with exercise training in the treatment of invasive breast cancer.

\section{Acknowledgements}

This work was supported by NCI R21 CA140916 (WCL).

\section{References}

1. Gleeson M, Bishop NC, Stensel DJ, Lindley MR, Mastana SS, et al. (2011) The anti-inflammatory effects of exercise: mechanisms and implications for the prevention and treatment of disease. Nat Rev Immunol 11: 607-615.

2. Petersen AM, Pedersen BK (2005) The anti-inflammatory effects of exercise. J Appl Physiol 98: 1154-1162.

3. Gomez-Cabrera MC, Domenech E, Vina J (2008) Moderate exercise is an antioxidant: upregulation of antioxidant genes by training. Free Rad Biol Med 44: 126-131.

4. Bailey RL, Gahche JJ, Lentino CV, Dwyer JT, Engel JS, et al. (2011) Dietary supplement use in the United States, 2003-2006. J Nutr 141: 261-266.
5. Patterson RE, Cadmus LA, Emond JA, Pierce JP (2010) Physical activity, diet adiposity and female breast cancer prognosis: a review of the epidemiologic literature. Maturitas 66: 5-15.

6. Holmes MD, Chen WY, Feskanich D, Kroenke CH, Colditz GA (2005) Physical activity and survival after breast cancer diagnosis. JAMA 293: 2479-2486.

7. Eliassen AH, Hankinson SE, Rosner B, Holmes MD, Willett WC (2010) Physical activity and risk of breast cancer among postmenopausal women. Arch Intern Med 170: 1758-1764

8. George SM, Irwin ML, Smith AW, Neuhouser ML, Reedy J, et al. (2011) Postdiagnosis diet quality, the combination of diet quality and recreational physical activity, and prognosis after early-stage breast cancer. Cancer Causes Control 22: 589-598.

9. Holick CN, Newcomb PA, Trentham-Dietz A, Titus-Ernstoff L, Bersch AJ, et al (2008) Physical activity and survival after diagnosis of invasive breast cancer. Cancer Epidemiol Biomarkers Prev 17: 379-386.

10. Zhu Z, Jiang W, Sells JL, Neil ES, McGinley JN, et al. (2008) Effects of nonmotorized wheel running on mammary carcinogenesis: circulating biomarkers, cellular processes, and molecular mechanisms in rats. Cancer Epidemiol Biomarkers Prev 17: 1920-1929.

11. Lin EY, Jones JG, Li P, Zhu L, Whitney KD, et al. (2003) Progression to malignancy in the polyoma middle $T$ oncoprotein mouse breast cancer mode provides a reliable model for human diseases. Am J Pathol 163: 2113-2126.

12. Powers SK, Jackson MJ (2008) Exercise-induced oxidative stress: cellular mechanisms and impact on muscle force production. Physiol Rev 88: 12431276.

13. Suvorava T, Kumpf S, Rauch BH, Dao VT, Adams V, et al. (2010) Hydrogen peroxide inhibits exercise-induced increase of circulating stem cells with endothelial progenitor capacity. Free Radic Res 44: 199-207.

14. Ji LL, Gomez-Cabrera M, Vina J (2006) Exercise and hormesis: activation of cellular antioxidant signaling pathway. Ann NY Acad Sci 1067: 425-435.

15. Weinberg RA (2008) Mechanisms of malignant progression. Carcinogenesis 29: 1092-1095

16. Radisky DC (2005) Epithelial-mesenchymal transition. J Cell Sci 118: 43254326.

17. Naik E, Dixit VM (2011) Mitochondrial reactive oxygen species drive proinflammatory cytokine production. J Exp Med 208: 417-420.

18. Demetrius LA, Coy JF, Tuszynski JA (2010) Cancer proliferation and therapy: the Warburg effect and quantum metabolism. Theor Biol Med Model 7: 2.

19. Weinberg F, Chandel NS (2009) Reactive oxygen species-dependent signaling regulates cancer. Cell Mol Life Sci 66: 3663-3673.

20. Belkhiri A, Richards C, Whaley M, McQueen SA, Orr FW (1997) Increased expression of activated matrix metalloproteinase-2 by human endothelial cells after sublethal $\mathrm{H} 2 \mathrm{O} 2$ exposure. Lab Invest 77: 533-539.

21. Treuting PM, Linford NJ, Knoblaugh SE, Emond MJ, Morton JF, et al. (2008) Reduction of age-associated pathology in old mice by overexpression of catalase in mitochondria. J Gerontol A Biol Sci Med Sci 63: 813-822.

22. Goh J, Enns L, Fatemie S, Hopkins H, Morton J, et al. (2011) Mitochondria targeted catalase suppresses invasive breast cancer in mice. BMC Cancer 11 191.

23. Lee HY, Choi CS, Birkenfeld AL, Alves TC, Jornayvaz FR, et al. (2010) Targeted expression of catalase to mitochondria prevents age-associated reductions in mitochondrial function and insulin resistance. Cell Metab 12: 688-674.

24. Lisanti MP, Martinez-Outschoorn UE, Pavlides S, Whitaker-Menezes D, Pestel RG, et al. (2011) Accelerated aging in the tumor microenvironment: connecting aging, inflammation and cancer metabolism with personalized medicine. Cell Cycle 10: 2059-2063.

25. Pollard JW (2008) Macrophages define the invasive microenvironment in breast cancer. J Leukoc Biol 84: 623-630.

26. Hagemann T, Lawrence T, McNeish I, Charles KA, Kulbe H, et al. (2008) "Re educating" tumor-associated macrophages by targeting NF-kappaB. J Exp Med 205: 1261-1268

27. Emtage PC, Clarke D, Gonzalo-Daganzo R, Junghans RP (2003) Generating potent Th1/Tc1 $\mathrm{T}$ cell adoptive immunotherapy doses using human IL-12: 
Citation: Goh J, Pettan-Brewer C, Enns L, Fatemie S, Ladiges W (2012) Are Exercise and Mitochondrial Antioxidants Compatible in the Treatment of Invasive Breast Cancer? Bioenerg Open Access 1:101. doi:10.4172/2167-7662.1000101

Harnessing the immunomodulatory potential of $\mathrm{IL}-12$ without the in vivoassociated toxicity. J Immunother 26: 97-106

28. Sica A, Bronte V (2007) Altered macrophage differentiation and immune dysfunction in tumor development. J Clin Invest 117: 1155-1166.

29. Biswas SK, Sica A, Lewis CE (2008) Plasticity of macrophage function during tumor progression: regulation by distinct molecular mechanisms. J Immunol 180: 2011-2017.

30. Woods JA, Lu Q, Ceddia MA, Lowder T (2000) Exercise-induced modulation of macrophage function. Immunol Cell Biol 78: 543-553.

31. Joyce JA, Pollard JW (2009) Microenvironmental regulation of metastasis. Nat Rev Cancer 9: 239-252.

32. Verma VK, Singh V, Singh MP, Singh SM (2009) Effect of physical exercise on tumor growth regulating factors of tumor microenvironment: Implications in exercise-dependent tumor growth retardation. Immunopharmacol Immunotoxicol 31: 274-282.

33. Pettan-Brewer C, Morton J, Mangalindan R, Ladiges W (2011) Curcumin suppresses intestinal polyps in APC Min mice fed a high fat diet. Pathobiology Aging and Age-related Dis 1: 7013- DOI:10.3402/pba.v1i0.7013.

34. Rogers CJ, Colbert LH, Greiner JW, Perkins SN, Hursting SD (2008) Physical activity and cancer prevention: pathways and targets for intervention. Sports Med 38: 271-296.

35. Ladiges W, Wanagat J, Preston B, Loeb L, Rabinovitch P (2010) A mitochondrial view of aging, reactive oxygen species and metastatic cancer. Aging Cell 9: 462-465
36. Davies KJ, Quintanilha AT, Brooks GA, Packer L (1982) Free radicals and tissue damage produced by exercise. Biochem Biophys Res Commun 107 1198-1205.

37. Jackson MJ (2008) Free radicals generated by contracting muscle: by products of metabolism or key regulators of muscle function? Free Radic Biol Med 44 132-141.

38. Navarro A, Boveris A (2007) The mitochondrial energy transduction system and the aging process. Am J Physiol Cell Physiol 292: 670-686.

39. McArdle F, Pattwell DM, Vailaki A, McArdle A, Jackson MJ (2005) Intracellula generation of reactive oxygen species by contracting skeletal muscle cells. Free Radic Biol Med 39: 651-657.

40. Xia R, Webb JA, Gnall LL, Cutler K, Abramson JJ (2003) Skeletal muscle sarcoplasmic reticulum contains a $\mathrm{NADH}$-dependent oxidase that generates superoxide. Am J Physiol Cell Physiol 285: C215-C221.

41. Gong MC, Arbogast S, Guo Z, Mathenia J, Su W, et al. (2006) Calcium independent phospholipase A2 modulates cytosolic oxidant activity and contractile function in murine muscle cells. J Appl Physiol 100: 399-405.

42. Sachdev S, Davies KJ (2008) Production, detection and adaptive responses to free radicals in exercise. Free Radic Biol Med 44: 215-233.

43. Min K, Smuder AJ, Kwon OS, Kavazis AN, Szeto HH, et al. (2011) Mitochondrialtargeted antioxidants protect the skeletal muscle against immobilizationinduced muscle atrophy. J Appl Physiol 111: 1459-1466.

44. Smith RA, Murphy MP (2010) Animal and human studies with the mitochondriatargeted antioxidant MitoQ. Ann N Y Acad Sci 1201: 96-103. 